Research Paper

\title{
Lower Body Mass Index is a Risk Factor for In-Hospital Mortality of Elderly Japanese Patients Treated with Ampicillin/sulbactam
}

Makoto Miura ${ }^{1 凶}$, Akiko Kuwahara ${ }^{2}$, Akinori Tomozawa ${ }^{3}$, Naoki Omae ${ }^{4}$, Motohiro Yamamori ${ }^{2}$, Kaori Kadoyama 5, and Toshiyuki Sakaeda 5,6凶

1. Department of Pharmacy, Rakuwakai Otowa Hospital, Kyoto 607-8062, Japan;

2. School of Pharmacy and Pharmaceutical Sciences, Mukogawa Women's University, Nishinomiya 663-8179, Japan;

3. Department of Pharmacy, Kyoto Kujo Hospital, Kyoto 601-8453, Japan;

4. Department of Pharmacy, Rakuwakai Marutamachi Hospital, Kyoto 604-8405, Japan;

5. Graduate School of Pharmaceutical Sciences, Kyoto University, Kyoto 606-8501, Japan;

6. Department of Pharmacokinetics, Kyoto Pharmaceutical University, Kyoto 607-8414, Japan.

$\triangle$ Corresponding authors: Toshiyuki Sakaeda, Ph.D., Department of Pharmacokinetics, Kyoto Pharmaceutical University, Kyoto 607-8414, Japan, Tel: +81-75-595-4625, Fax: +81-75-595-4751, e-mail: sakaedat@mb.kyoto-phu.ac.jp; Makoto Miura, M.Sc., Department of Pharmacy, Rakuwakai Otowa Hospital, Kyoto 607-8062, Japan, Tel: +81-75-593-6186, Fax: +81-75-593-9200, e-mail: miura_m@rakuwa.or.jp.

(C) Ivyspring International Publisher. Reproduction is permitted for personal, noncommercial use, provided that the article is in whole, unmodified, and properly cited. See http://ivyspring.com/terms for terms and conditions.

Received: 2016.05.07; Accepted: 2016.08.22; Published: 2016.09.19

\begin{abstract}
Objectives: A retrospective examination was conducted to identify risk factors for in-hospital mortality of elderly patients (65 years or older) treated with the beta-lactam/beta-lactamase inhibitor combination antibiotic, ampicillin/sulbactam (ABPC/SBT).

Methods: Clinical data from 96 patients who were hospitalized with infectious diseases and treated with ABPC/SBT ( $9 \mathrm{~g} /$ day or $12 \mathrm{~g} /$ day) were analyzed. Risk factors examined included demographic and clinical laboratory parameters. Parameter values prior to treatment and changes after treatment were compared between survivors and non-survivors.

Results: The study patients had an average age of $81.9 \pm 8.4$ years $( \pm S D)$ and body mass index (BMI) of $19.9 \pm 4.2 \mathrm{~kg} / \mathrm{m}^{2}$. They were characterized by anemia (low hemoglobin and hematocrit levels), inflammation (high leukocyte count, neutrophil count, C-reactive protein level, and body temperature), and hepatic and renal dysfunction (high aspartate aminotransferase, alanine aminotransferase and blood urea nitrogen levels). The BMI of non-survivors, $16.2 \pm 2.9 \mathrm{~kg} / \mathrm{m}^{2}$, was lower than that of survivors, $20.4 \pm 4.1 \mathrm{~kg} / \mathrm{m}^{2}$. In addition, the hematological parameters deteriorated more remarkably, inflammation markers were not altered (or the decrease was marginal), and hepatic function was not improved, in non-survivors.
\end{abstract}

Conclusions: A lower BMI value is a risk factor for in-hospital mortality of elderly patients treated with $\mathrm{ABPC} / \mathrm{SBT}$.

Key words: ampicillin/sulbactam, elderly patients, mortality, body mass index.

\section{Introduction}

Generally, elderly patients, usually defined as age 65 years or older, are often weak, undernourished, and hypokinetic, with delirium/dementia. They can also present a sudden change in motor function (e.g., balance impairment) when compared with younger patients. These characteristics are often described by the term "frail" [1]. Frailty has been recognized as an important factor influencing the prognosis of diseases after treatments [1]. However, there is no clinical indicator of frailty [1]. Elderly patients also show a higher prevalence of several diseases including diabetes, chronic renal or hepatic failure, congestive heart failure, malignancy, chronic pulmonary diseases, and infectious diseases. These diseases can 
adversely affect treatment outcomes.

Pneumonia is one of main causes of mortality in the elderly. In terms of severity of symptoms, the clinical presentation of pneumonia can be quite different from that observed in younger patients [1]. Microbiological patterns among elderly patients with community-acquired pneumonia (CAP) have been shown to be different from those of younger patients $[2,3]$. These patterns in the elderly include higher rates of pneumococcal and influenza viral pneumonia, and lower presence of atypical pathogens $[2,3]$. Elderly patients are also susceptible to multi-drug resistant pathogens, and they present more risk factors for aspiration pneumonia [1]. Epidemiological investigations suggest an increased tendency to infections, but this is not explained by suppression of immunological reactions [4].

Ampicillin/sulbactam (ABPC/SBT) is a beta-lactam/beta-lactamase inhibitor combination (dose ratio of 2:1) with broad spectrum of antibacterial activity against Gram-positive, Gram-negative and anaerobic bacteria [5]. It is used for treatment of lower respiratory tract infections and aspiration pneumonia, gynaecological/obstertrical infections, intraabdominal infections, pediatric infections, diabetic foot infections and skin and soft tissue infections [5]. According to several international guidelines for the treatment of CAP published in 2007 to 2012 [6-8], ABPC/SBT $(9 \mathrm{~g} /$ day at $3 \mathrm{~g}$ every $8 \mathrm{hr}$ ) is recommended for hospitalized patients with non-severe pneumonia [9]. The approved maximal daily dose of ABPC/SBT is $12 \mathrm{~g}$ ( $3 \mathrm{~g}$ every $6 \mathrm{hr}$ ) in many countries. However, in Japan, it was recently shown that $12 \mathrm{~g} /$ day is well tolerated and provides excellent clinical and bacteriological responses [10]; this dose was approved in 2012.

In 2003, an international multicenter study was conducted in order to establish a practical severity assessment model for stratifying adults hospitalized with CAP into different management groups [11]. It was demonstrated that an age of 65 years or older was a risk factor for mortality [11]. In the present study, a retrospective examination was conducted to identify risk factors for in-hospital elderly patients, aged 65 years or older, who were treated with ABPC/SBT. Changes after treatment were also compared between survivors and non-survivors to obtain additional information that could be used to more effectively manage elderly patients.

\section{Patient and Methods}

\section{Eligibility}

All elderly patients (65 years or older) with infectious diseases were hospitalized and treated with
$9 \mathrm{~g} /$ day or $12 \mathrm{~g} /$ day of ABPC/SBT at Rakuwakai Otowa Hospital, Japan from December 2011 to July 2012. Patients with the following conditions were excluded: 1) liver or renal dysfunctions (CTCAE [Common Terminology Criteria for Adverse Events] ver.4 grade 3 or higher), 2) undergoing cancer chemotherapy, 3) cardiopulmonary resuscitation, 4) ICU admission during hospitalization, or 5) operation during hospitalization. This retrospective study was approved by the ethics committee at Rakuwakai Otowa Hospital, Japan.

\section{Data analysis}

Patients were classified into two groups based on the mortality; i.e., survivors and non-survivors. Risk factors examined included age, gender, body weight, height, body mass index (BMI), and daily dose and number of doses of ABPC/SBT. Measurements of the following clinical laboratory parameters were made one day before, or on the day of, the start of treatment; erythrocyte count, hemoglobin, hematocrit, leukocyte count, neutrophil count, lymphocyte count, eosinophil count, basophil count, monocyte count, platelet count, aspartate aminotransferase (AST), alanine aminotransferase (ALT), gamma-glutamyl transpeptidase ( $\gamma$-GTP), total bilirubin (T-bil), alkaline phosphatase (ALP), lactate dehydrogenase (LDH), blood urea nitrogen (BUN), serum creatinine (Scr), C-reactive protein (CRP), and body temperature. These were also measured one day after the treatment. In cases where treatment ended within 3 days, the data were not included. If the patients died within 7 days after the last clinical laboratory test, the data were also excluded.

\section{Statistical analysis}

All values were reported as mean \pm standard deviations (SD). The unpaired Student's t-test/Welch`s test or Mann-Whitney`s U test was used for two-groups comparisons of values. Fisher's exact test was used for the analysis of contingency tables. $\mathrm{P}$ values of less than 0.05 were considered significant.

\section{Results}

Data from 96 patients were analyzed. Demographics, daily doses and number of doses of ABPC/SBT are summarized in Table 1. Clinical diagnoses included aspiration pneumonia $(\mathrm{N}=27)$, pneumonia $(\mathrm{N}=13)$, urinary tract infection $(\mathrm{N}=8)$, acute cholecystitis $(\mathrm{N}=6)$, acute cholangitis $(\mathrm{N}=5)$, choledocholithiasis cholangitis $(\mathrm{N}=5), \quad$ acute pyelonephritis $(\mathrm{N}=4)$, complicated urinary tract infection $(\mathrm{N}=3)$, acute pneumonia $(\mathrm{N}=2)$ and others $(\mathrm{N}=23)$. Eighty-three patients $(86.5 \%)$ were survivors, 
whereas 13 were died after treatment. The causes of death included senility $(\mathrm{N}=3)$, aspiration pneumonia $(\mathrm{N}=2)$ and others $(\mathrm{N}=8)$. The average values for age, body weight and height were $81.9 \pm 8.4$ years, $47.6 \pm 11.8$ $\mathrm{kg}$, and $154.1 \pm 9.9 \mathrm{~cm}$, respectively (Table 1). There were no statistical differences between survivors and non-survivors. However, the BMI of non-survivors, $16.2 \pm 2.9 \mathrm{~kg} / \mathrm{m}^{2}$, was significantly lower than that of survivors, $20.4 \pm 4.1 \mathrm{~kg} / \mathrm{m}^{2}$.

Table 2 shows values for 12 of the 20 clinical laboratory parameters measured prior to treatment of patients with ABPC/SBT. Patients were characterized by anemia (low hemoglobin and hematocrit levels), inflammation (high leukocyte count, neutrophil count, CRP level, and body temperature), and hepatic and renal dysfunction (high AST, ALT and BUN levels). No meaningful differences in clinical laboratory parameters were observed between survivors and non- survivors, except for neutrophil count and BUN. The neutrophil count for non-survivors was $7.2 \pm 4.8 \times 10^{3} / \mu \mathrm{L}$, which was significantly lower than that for the survivors, $10.0 \pm 5.6 \times 10^{3} / \mu \mathrm{L}$. The BUN value for non-survivors was $31.3 \pm 28.3 \mathrm{mg} / \mathrm{dL}$, which was significantly higher than that for the survivors, $20.5 \pm 13.4 \mathrm{mg} / \mathrm{dL}$. There were no differences between survivors and non-survivors in the other 8 clinical laboratory parameters (data not shown).

Values of clinical laboratory parameters measured after treatment with ABPC/SBT are summarized in Table 3. Non-survivors showed significantly lower values for erythrocyte count, hemoglobin, hematocrit and platelet count, and higher values for BUN and CRP than survivors. Changes after treatment with ABPC/SBT are shown in Table 4. The values for erythrocyte count, hemoglobin and hematocrit deteriorated more remarkably in non-survivors. Leukocyte and neutrophil counts were not changed, and levels of AST and ALT were not improved in non-survivors.

Table 1. Characteristics of patients prior to treatment with ampicillin/sulbactam

\begin{tabular}{llll}
\hline & Total & Survivors & Non-survivors \\
\hline Number of patients & 96 & 83 & 13 \\
Age, years & $81.9 \pm 8.4$ & $81.6 \pm 8.5$ & $84.2 \pm 7.8$ \\
Gender, male/female & $44 / 52$ & $36 / 47$ & $8 / 5$ \\
Body weight, kg & $47.6 \pm 11.8$ & $48.4 \pm 11.7$ & $40.5 \pm 9.8$ \\
Height, cm & $154.1 \pm 9.9$ & $153.8 \pm 9.7$ & $156.1 \pm 11.5$ \\
Body mass index, kg/m2 & $19.9 \pm 4.2$ & $20.4 \pm 4.1$ & $16.2 \pm 2.9 *$ \\
Dose, g/day & $9.8 \pm 2.1$ & $9.8 \pm 2.1$ & $9.7 \pm 2.2$ \\
Number of doses, /day & $3.3 \pm 0.6$ & $3.3 \pm 0.6$ & $3.2 \pm 0.7$
\end{tabular}

Values are mean \pm standard deviations

${ }^{*} \mathrm{p}<0.05$, compared with survivors.
Table 2. Clinical laboratory parameters for patients prior to treatment with ampicillin/sulbactam.

\begin{tabular}{llll}
\hline & Total & Survivors & Non-survivors \\
\hline Erythrocyte count, $\times 10^{6} / \mu \mathrm{l}$ & $4.1 \pm 0.7$ & $4.1 \pm 0.7$ & $3.8 \pm 0.8$ \\
Hemoglobin, $\mathrm{g} / \mathrm{dL}$ & $12.4 \pm 2.2$ & $12.5 \pm 2.2$ & $11.4 \pm 2.5$ \\
Hematocrit, $\%$ & $37.1 \pm 6.4$ & $37.5 \pm 6.1$ & $34.8 \pm 7.8$ \\
Leukocyte count, $\times 10^{3} / \mu \mathrm{l}$ & $11.0 \pm 5.8$ & $11.4 \pm 5.8$ & $8.6 \pm 5.2$ \\
Neutrophil count, $\times 10^{3} / \mu \mathrm{l}$ & $9.6 \pm 5.6$ & $10.0 \pm 5.6$ & $7.2 \pm 4.8$ * \\
Platelet count, $\times 10^{3} / \mu \mathrm{l}$ & $201.3 \pm 82.4$ & $202.9 \pm 77.1$ & $191.1 \pm 114.4$ \\
AST, U/L & $78.6 \pm 204.6$ & $86.6 \pm 219.1$ & $27.2 \pm 10.2$ \\
ALT, U/L & $49.5 \pm 84.8$ & $53.9 \pm 89.9$ & $19.8 \pm 11.6$ \\
BUN, mg/dL & $22.0 \pm 16.4$ & $20.5 \pm 13.4$ & $31.3 \pm 28.3$ * \\
Serum creatinine, $\mathrm{mg} / \mathrm{dL}$ & $0.9 \pm 0.9$ & $0.9 \pm 0.9$ & $1.0 \pm 0.8$ \\
CRP, mg/dL & $7.7 \pm 7.5$ & $7.8 \pm 7.8$ & $7.3 \pm 6.2$ \\
Body temperature, degrees & $37.6 \pm 0.8$ & $37.6 \pm 0.8$ & $37.5 \pm 0.9$
\end{tabular}

Values are mean \pm standard deviations.

${ }^{*} \mathrm{p}<0.05$, compared with survivors

Table 3. Clinical laboratory parameters for patients after treatment with ampicillin/sulbactam.

\begin{tabular}{llll}
\hline & Total & Survivors & Non-survivors \\
\hline Erythrocyte count, $\times 10^{6} / \mu \mathrm{l}$ & $3.7 \pm 0.6$ & $3.8 \pm 0.6$ & $3.1 \pm 0.6^{*}$ \\
Hemoglobin, $\mathrm{g} / \mathrm{dL}$ & $11.1 \pm 1.9$ & $11.4 \pm 1.7$ & $9.3 \pm 1.7{ }^{*}$ \\
Hematocrit, \% & $33.9 \pm 5.5$ & $34.7 \pm 5.0$ & $28.3 \pm 5.3$ * \\
Leukocyte count, $\times 10^{3} / \mu \mathrm{l}$ & $6.3 \pm 3.2$ & $6.0 \pm 2.0$ & $8.5 \pm 6.8$ \\
Neutrophil count, $\times 10^{3} / \mu \mathrm{l}$ & $4.5 \pm 3.2$ & $4.1 \pm 1.9$ & $7.2 \pm 6.8$ \\
Platelet count, $\times 10^{3} / \mu \mathrm{l}$ & $237.3 \pm 88.2$ & $246.0 \pm 84.0$ & $181.8 \pm 97.2^{*}$ \\
AST, U/L & $31.0 \pm 21.3$ & $30.2 \pm 20.7$ & $35.6 \pm 25.3$ \\
ALT, U/L & $28.3 \pm 26.9$ & $28.9 \pm 28.5$ & $24.9 \pm 14.5$ \\
BUN, mg/dL & $13.1 \pm 15.4$ & $11.2 \pm 7.2$ & $24.8 \pm 36.8$ * \\
Scr, mg/dL & $0.7 \pm 0.4$ & $0.7 \pm 0.4$ & $0.8 \pm 0.4$ \\
CRP, mg/dL & $2.8 \pm 3.6$ & $2.5 \pm 3.7$ & $4.3 \pm 2.4 *$ \\
Body temperature, degrees & $36.8 \pm 0.4$ & $36.8 \pm 0.4$ & $36.7 \pm 0.5$
\end{tabular}

Values are mean \pm standard deviations.

${ }^{*} \mathrm{p}<0.05$, compared with survivors.

Table 4. Changes in clinical laboratory parameters after treatment with ampicillin/sulbactam.

\begin{tabular}{llll}
\hline & Total & Survivors & Non-survivors \\
\hline Erythrocyte count, $\times 10^{6} / \mu \mathrm{l}$ & $-0.4 \pm 0.4$ & $-0.3 \pm 0.4$ & $-0.7 \pm 0.4$ * \\
Hemoglobin, g/dL & $-1.3 \pm 1.3$ & $-1.1 \pm 1.2$ & $-2.1 \pm 1.2$ * \\
Hematocrit, $\%$ & $-3.2 \pm 4.0$ & $-2.7 \pm 3.7$ & $-6.5 \pm 4.0$ * \\
Leukocyte count, $\times 10^{3} / \mu \mathrm{l}$ & $-4.7 \pm 6.3$ & $-5.4 \pm 5.7$ & $0.0 \pm 8.3$ * \\
Neutrophil count, $\times 10^{3} / \mu \mathrm{l}$ & $-5.1 \pm 6.5$ & $-5.9 \pm 5.7$ & $0.0 \pm 8.3$ * \\
Platelet count, $\times 10^{3} / \mu \mathrm{l}$ & $36.0 \pm 76.1$ & $43.0 \pm 70.6$ & $-9.2 \pm 96.0$ * \\
AST, U/L & $-48.4 \pm 208.5$ & $-57.8 \pm 223.7$ & $8.5 \pm 19.9$ \\
ALT, U/L & $-21.2 \pm 76.9$ & $-25.4 \pm 81.5$ & $6.8 \pm 11.4$ * \\
BUN, mg/dL & $-8.9 \pm 19.5$ & $-9.3 \pm 12.7$ & $-6.5 \pm 43.5$ \\
Scr, mg/dL & $-0.1 \pm 0.6$ & $-0.1 \pm 0.6$ & $-0.2 \pm 0.7$ \\
CRP, mg/dL & $-5.1 \pm 8.1$ & $-5.4 \pm 8.4$ & $-3.1 \pm 6.3$ \\
Body temperature, degrees & $-0.7 \pm 1.0$ & $-0.7 \pm 1.0$ & $-0.8 \pm 0.9$ \\
\hline
\end{tabular}

Values are mean \pm standard deviations.

${ }^{*} \mathrm{p}<0.05$, compared with survivors.

\section{Discussion}

In this study, it was demonstrated that a lower BMI was a risk factor for in-hospital mortality of elderly patients treated with ABPC/SBT (Table 1). Recently, a large-scale meta-analysis on the 
association of BMI with all-cause mortality was published [12]. The sample size was over 2.88 million individuals and it included 270,000 deaths. Results showed that the mortality was lower in overweight (BMI $25-29.9 \mathrm{~kg} / \mathrm{m}^{2}$ ), similar in grade 1 obesity (BMI $30-34.9 \mathrm{~kg} / \mathrm{m}^{2}$ ), and higher in grade $2 / 3$ obesity (BMI $35 \mathrm{~kg} / \mathrm{m}^{2}$ or more), when compared with normal (BMI 18.5-24.9 kg/m²) [12]. Soon after this report, another study was published based on a sample size of over 70,000 elderly people [13]. This study demonstrated that underweight (BMI $<18.5 \mathrm{~kg} / \mathrm{m}^{2}$ ) showed a higher mortality than normal BMI [13]. Taken together, these studies show that the association between BMI and mortality is U-shaped, with overweight or grade 1 obesity being at the minimum: this is the so-called 'obesity paradox' [14].

On the other hand, there are several reports showing a positive correlation between the BMI and the prevalence of hypertension and diabetes $[15,16]$. The effect of triglycerides on coronary heart disease has also shown to be dependent on the BMI [17]. Bo et al. [18] suggested that the prognosis of 659 elderly patients admitted to medical intensive care units depended not only on the severity and age, but also on preexisting conditions. These conditions included loss of functional independence, severe and moderate cognitive impairment, and low BMI; higher mortality was found in the patients with lower BMI [18].

In our study, mean BMI values were $20.4 \mathrm{~kg} / \mathrm{m}^{2}$ and $16.2 \mathrm{~kg} / \mathrm{m}^{2}$ for survivors and non-survivors, respectively. This results is consistent with the 'obesity paradox' [14] and the report by Bo et al [18]. Other risk factors for mortality from our study included relatively low neutrophil count and high BUN (Table 2). Little information is available on mortality associated with neutrophil count, but renal impairment is a well-recognized risk factor for mortality. Further extensive examination with a large number of patients is needed to clarify the mortality predictability of clinical laboratory parameters.

Treatment with ABPC/SBT had no effects on hematological parameters (erythrocyte count, and hemoglobin and hematocrit values) in survivors (Table 4). However, treatment did cause a decrease in these parameters in non-survivors (Table 4). Values for inflammation markers (leukocyte and neutrophil counts, and CRP value) were decreased for survivors, but not for non-survivors, values were either not altered or decrease was marginal (Table 4). Treatment with ABPC/SBT attenuated hepatic dysfunction in survivors, but no such attenuation was observed for non-survivors (Table 4).

Adverse effects associated with ABPC/SBT include hematological abnormalities [5], and the mortality may be, at least in part, due to these effects.
$\mathrm{ABPC} / \mathrm{SBT}$ is primarily eliminated by renal excretion [5], and its pharmacokinetics depends on renal function [19-22]. In our study, values of Scr for all patients were within the normal range prior to treatment with ABPC/SBT (Table 2); therefore, we did not modify the dose. However, creatinine is formed in muscle tissues as a break-down product of creatine, and its formation is affected by age, gender, race, habits, diet, and chronic diseases [23]. As non-survivors showed a relatively low BMI, it is possible that the renal function was incorrectly estimated. As such, $9 \mathrm{~g} /$ day or $12 \mathrm{~g} /$ day ABPC/SBT had to be considered as a potential overdose for these patients.

Craig and his co-workers [24] have conducted a series of investigations on the interrelationships between the pharmacokinetics and pharmacodynamics of a variety of antibiotics. They divided these antibiotics into 3 categories based on the pharmacokinetic parameters that determined efficacy. The efficacy of beta-lactam antibiotics, including $\mathrm{ABPC} / \mathrm{SBT}$, depends on the percentage of time that plasma concentrations exceed the minimum inhibitory concentration against the causative pathogen during the dosing interval. Thus, $12 \mathrm{~g} /$ day (3 g every $6 \mathrm{hr}$ ) of ABPC/SBT is assumed to be superior to $9 \mathrm{~g} /$ day ( $3 \mathrm{~g}$ every $8 \mathrm{hr}$ ) in terms of efficacy. However, at present there is a lack of comparative data, especially for elderly patients, to make any definitive conclusion. Therefore, in this study, we divided patients into 2 groups based on the ABPC/SBT daily dose. No statistical differences between these 2 groups were observed in the clinical laboratory parameters or in mortality (data not shown). Our data suggested that $12 \mathrm{~g} /$ day of $\mathrm{ABPC} / \mathrm{SBT}$ was as well tolerated as $9 \mathrm{~g} /$ day. This result is similar to findings from earlier study [10].

Our study has limitations. It addresses only all-cause mortality and not morbidity or cause-specific mortality. A variety of infectious diseases required the prescription of $\mathrm{ABPC} / \mathrm{SBT}$, but culture tests showed no micro-organisms after the treatment in all patients, suggesting that the mortality was not due to the lack of efficacy. High CRP values after the treatment with ABPC/SBT suggested chronic inflammation which was not related to infectious diseases. Especially for elderly patients, pre-existing conditions, including the frailty, affect the prognosis [1], but further analysis was limited by a small sample size.

In conclusion, a lower BMI was a risk factor for in-hospital mortality of elderly patients treated with ABPC/SBT. In non-survivors, the hematological parameters deteriorated more remarkably, inflammation markers were not altered (or the 
decrease was marginal), and hepatic function was not improved.

\section{Conflict of Interest}

The authors have declared that no conflict of interest exists.

\section{References}

1. Faverio $P$, Aliberti S, Bellelli G, et al. The management of community-acquired pneumonia in the elderly. Eur.J.Intern.Med. 2014; 25: 312-319.

2. Klapdor B, Ewig S, Pletz MW, et al. Community-acquired pneumonia in younger patients is an entity on its own. Eur.Respir.J. 2012; 39: 1156-1161.

3. Fung HB and Monteagudo-Chu MO. Community-acquired pneumonia in the elderly. Am.J.Geriatr.Pharmacother. 2010; 8: 47-62.

4. Dewan SK, Zheng SB, Xia SJ, et al. Senescent remodeling of the immune system and its contribution to the predisposition of the elderly to infections. Chin.Med.J. 2012; 125: 3325-3331.

5. Rafailidis PI, Ioannidou EN, Falagas ME. Ampicillin/sulbactam current status in severe bacterial infections. Drugs. 2007; 67: 1829-1849.

6. Mandell LA, Wunderink RG, Anzueto A, et al. Infectious Diseases Society of America/American Thoracic Society consensus guidelines on the management of community-acquired pneumonia in adults. Clin.Infect.Dis. 2007; 44: S27-72.

7. Lim WS, Baudouin SV, George RC, et al. BTS guidelines for the management of community acquired pneumonia in adults: update 2009. Thorax. 2009; 64: iii1-55.

8. Spindler C, Strålin K, Eriksson L, et al. Swedish guidelines on the management of community-acquired pneumonia in immunocompetent adults -Swedish Society of Infectious Diseases 2012. Scand.J.Infect.Dis. 2012; 44: 885-902.

9. Thiem U, Heppner HJ, Pientka L. Elderly patients with community-acquired pneumonia: optimal treatment strategies. Drugs Aging. 2011; 28: 519-537.

10. Kohno S, Tateda K, Mikamo H, et al. Efficacy and safety of intravenous sulbactam/ampicillin $3 \mathrm{~g} 4$ times daily in Japanese adults with moderate to severe community-acquired pneumoniae: a multicenter, open-label, uncontrolled study. J.Infect.Chemother. 2015; 21: 182-188.

11. Lim WS, van der Eerden MM, Laing R, et al. Defining community acquired pneumonia severity on presentation to hospital: an international derivation and validation study. Thorax. 2003; 58: 377-382.

12. Flegal KM, Kit BK, Orpana $\mathrm{H}$, et al. Association of all-cause mortality with overweight and obesity using standard body mass index categories: a systematic review and meta-analysis. JAMA. 2013; 309: 71-82.

13. Wu C-Y, Chou YC, Huang N, et al. Association of body mass index with all-cause and cardiovascular disease mortality in the elderly. PLoS One. 2014; 9: e102589.

14. Dixon JB, Egger GJ, Finkelstein EA, et al. 'Obesity paradox' misunderstands the biology of optimal weight throughout the life cycle. Int.J.Obes (Lond). 2015; 39: 82-84.

15. Brown CD, Higgins M, Donato KA, et al. Body mass index and the prevalence of hypertension and dyslipidemia. Obes.Res. 2000; 8: 605-619.

16. Chan JM, Rimm EB, Colditz GA, et al. Obesity, fat distribution, and weight gain as risk factors for clinical diabetes in men. Diabetes Care. 1994; 17: 961-969.

17. Hirakawa $\mathrm{Y}, \mathrm{Lam} \mathrm{TH}$, Welborn $\mathrm{T}$, et al. The impact of body mass index on the associations of lipids with the risk of coronary heart disease in the Asia Pacific region. Prev.Med.Rep. 2015; 3: 79-82.

18. Bo M, Massaia M, Raspo S, et al. Predictive factors of in-hospital mortality in older patients admitted to a medical intensive care unit. J.Am.Geriatr.Soc. 2003; 51: 529-533.

19. Rho JP, Jones A, Woo M, et al. Single-dose pharmacokinetics of intravenous ampicillin plus sulbactam in healthy elderly and young adult subjects. J.Antimicrob.Chemother. 1989; 24: 573-580.

20. Meyers BR, Wilkinson $\mathrm{P}$, Mendelson $\mathrm{MH}$, et al. Pharmacokinetics of ampicillin/sulbactam in healthy elderly and young volunteers. Antimicrob.Agents Chemother. 1991; 35: 2098-2101.

21. Soto E, Shoji S, Muto C, et al. Population pharmacokinetics of ampicillin and sulbactam in patients with community-acquired pneumonia: evaluation of the impact of renal impairment. Br.J.Clin.Pharmacol. 2014; 77: 509-521.

22. Majcher-Peszynska J, Loebermann M, Klammt S, et al. Ampicillin/sulbactam in elderly patients with community-acquired pneumonia. Infection. 2014; 42: 79-87.

23. Stevens LA, Coresh J, Greene T, et al. Assessing kidney function--measured and estimated glomerular filtration rate. N.Engl.J.Med. 2006; 354: 2473-2483.

24. Craig WA. Pharmacokinetic/pharmacodynamics parameters: rationale for antibacterial dosing of mice and men. Clin.Infect.Dis. 1997; 26: 1-12. 